\title{
PER L'EUTANASIA
}

Note minime sul diritto a decidere della vita e della salute*

Riccardo Conte

SOMMARIO: 1. Introduzione e premesse (con una nota su Kant) - 2. Posizione del magistero ecclesiastico - 3. Note sul pensiero cristiano minoritario 4. La legge olandese sull'eutanasia - 5. La legge italiana - 6. La giurisprudenza della Corte europea dei diritti dell'uomo - 7. Il caso Welby - 8. Progetti di legge italiani e conclusione.

\section{Introduzione e premesse (con una nota su Kant)}

Discutere delle tematiche di fine vita è un compito difficile, anche per chi, come me, ha profonde, radicate convinzioni relative alla libertà della scelta. Da tempo ho depositato il mio testamento biologico, grazie all'iniziativa promossa a Milano dalla Chiesa valdese. Eppure quando mi accingo a parlare di queste tematiche, anche in privato, non lo faccio «a cuor leggero». Mi sono spesso imbattuto in persone che rispetto ad esse provano un rifiuto assoluto, o per motivi ideologici, o per motivi strettamente personali.

Io parto da una premessa ben chiara e, a mio modo di vedere, indiscutibile: siamo esseri umani liberi. Non so - pur avendo un background culturale cristiano - da dove vengo e dove vado. Non so se sono su questa terra per una volontà divina o per caso. Ma so che - grazie ad una storia di secoli di lotte sono un uomo libero. Ho libertà di parola, ho libertà di associazione, ho libertà di culto, posso decidere se sposarmi o no, ecc. Sono tutti diritti che trovano riconoscimento nelle Costituzioni degli Stati democratici e in Convenzioni internazionali, in primo luogo quella dei Diritti dell'Uomo. Ma oggi possiamo dire che vi è un'ulteriore consapevolezza. Infatti, l'art. 8 della Convenzione europea dei Diritti dell'Uomo - che non solo è legge dello Stato italiano, ma ha una particolare valenza superiore alle leggi ordinarie ${ }^{1}$ - dispone:

1. Ogni persona ha diritto al rispetto della sua vita privata e familiare, del suo domicilio e della sua corrispondenza. 2. Non può esservi ingerenza della pubblica autorità nell'esercizio di tale diritto a meno che tale ingerenza sia prevista dalla legge e costituisca una misura che, in una società democratica, è necessaria per la sicurezza nazionale, l'ordine pubblico, il benessere economico del paese, la prevenzione dei reati, la protezione della salute o della morale, o la protezione dei diritti e delle libertà altrui.

E la giurisprudenza della Corte europea dei diritti dell'Uomo ha ormai affermato (nel 2011) che «il diritto di un individuo a decidere in quale maniera e in quale momento la sua vita debba essere chiusa, a condizione che egli sia in grado di formare liberamente la sua volontà e il suo proposito d'agire in conse-

\footnotetext{
* L'articolo, con l'aggiunta di note bibliografiche minimali, riproduce il testo di una relazione svolta dall'Autore al Convegno dei Giuristi Democrati tenutosi a Milano il 19 gennaio 2013 sul tema «Lo stato e le prospettive dei diritti civili. Il ruolo propulsivo degli enti locali».

${ }^{1}$ Cfr. sul punto Corte cost., 24 ottobre 2007, n. 348, in «Corriere Giuridico», 2008, pp. 185 ss., spec. p. 187 con note di M. Luciani e R. Conti.
} 
guenza, è uno degli aspetti della sua vita privata ai sensi dell'art. 8 della Convenzione» ${ }^{2}$.

Non capisco, infatti, davvero perché non potrei avere la libertà di decidere se vivere o no, di decidere se la vita val la pena di essere vissuta, specialmente laddove io fossi convinto - per questioni di malattia, vuoi fisica, vuoi psichica - che la mia vita (la mia vita!) non fosse più degna di essere vissuta. Decisione su di me - sia chiaro! - non su un altro! Opporsi a questa visione affermando che della mia vita io non sono padrone, perché essa è un dono divino, che mi fa partecipare del suo soffio vitale (cfr. Enciclica Evangelium vitae di Giovanni Paolo II, \39) è affermazione che presuppone la condivisione di un discorso di fede ${ }^{3}$. Ma se io non condivido quel discorso di fede, non mi si possono imporre determinate scelte.

Prima di procedere in questo discorso, desidero fare una premessa. In questi anni vi sono state tante discussioni, troppe, sulla morte di Eluana Englaro, Piergiorgio Welby, Terry Schiavo, ecc. Raramente discussioni prudenti, rispettose delle persone e del dolore. Discussioni per lo più vergognose. Iniziative vergognose. Analogamente vi sono state tante discussioni, troppe, sulla morte di Giovanni Paolo II e, recentemente, del Card. Martini. Io mi guarderò

${ }^{2}$ Corte europea dei diritti dell'uomo, 20 gennaio 2011, Haas v. Suisse ( $(51)$.

${ }^{3} \mathrm{Vi}$ è una sorta di fil ronge, o, dato il tema, di fil noire, che attraversa i secoli, unendo il pensiero di condanna del suicidio. Aristotele affermava che «una specie di pubblica infamia colpisce chi si uccide, in quanto commette ingiustizia contro la città» (ARISTOTELE, Etica Nicomachea, V, 11, 1138a15. Il passo è richiamato da Tommaso d'Aquino, La somma teologica, II-II, q. 64, a. 5, nel paragrafo Respondeo). Lenin, in un'intervista specificamente dedicata al suicidio dopo le esequie del genero e della figlia di Marx nel novembre del 1911, formulò così la sua opinione: «Un socialista non appartiene a sé stesso, ma al partito. Se può in qualche modo essere ancora utile alla classe operaia, per esempio con lo stendere se non altro un articolo o un proclama, non ha il diritto di suicidarsi». Paul Lafargue si suicidò nella notte tra il 26 ed il 27 novembre 1911, insieme alla moglie Laura Marx. Fra alcune lettere che lasciò se ne trovò una in cui si legge: «Sano di corpo e di spirito, mi uccido prima che l'impietosa vecchiaia mi tolga uno a uno i piaceri e le gioie dell'esistenza e mi spogli delle forze fisiche e intellettuali. // Affinché la vecchiaia non paralizzi la mia energia, non spezzi la mia volontà e non mi renda un peso per me e per gli altri. // Da molto tempo mi sono ripromesso di non superare i settant'anni». Gli mancavano, infatti, meno di tre mesi per compierli. Non voglio certamente opporre a questo pensiero quello forse troppo radicale di David Hume, Sul suicidio (cfr. AA.VV. In difesa dell'eutanasia, a cura di C. Angelino, il melangolo, Genova 2007, pp. 50 ss.) sul punto: «Supponete che io non sia più in grado di agire nell'interesse della società; supponete che sia soltanto un peso; supponete che la mia vita sia soltanto un peso; supponete che la mia vita impedisca ad altre persone di essere utili alla società. In tali casi la mia rinunzia alla vita può essere non solo innocente, ma lodevole». Il problema non è il peso sulla società: noi siamo arrivati a costituzionalizzare il principio di solidarietà. Il problema, semmai, è il peso per il soggetto che soffre della sua malattia. Sul punto vorrei ricordare Seneca, Sulla morte volontaria (Lettere a Lucilio, VIII, 70), cfr. In difesa dell'eutanasia, cit. , pp. 27 ss.: «La vita [...] non sempre merita di essere conservata. Non è un bene il vivere, ma il vivere bene»; e poco più avanti: «L'importante non è morire più presto o più tardi, ma morire bene o male. Ora, morire bene significa sfuggire al pericolo di vivere male», talché è un nonsenso affermare «finché c'è vita c'è speranza». E ancora: «Se uno ha una scelta tra una morte in mezzo ai tormenti e una morte senza sofferenze, perché non dovrebbe scegliere quest'ultima?»; infatti, «non è vero che una vita più lunga è sempre la migliore, è vero che è sempre la peggiore una morte che si prolunga». Dal canto suo, ne Le leggi, Platone prevede particolari sanzioni per il suicida (sepoltura in luogo isolato, senza stele), ma non considera illegittimo il suicidio laddove vi sia un ordine della città (per una condanna), oppure perché «costretto da una sciagura molto dolorosa che si è abbattuta inevitabile su di lui [...], [oppure per] qualche onta insuperabile ed insopportabile» (Platone, Le leggi, IX, $873 \mathrm{c}-\mathrm{d}$ ). 
bene qui dallo scendere su un piano che trovo davvero poco rispettoso. La morte è un momento particolarissimo della vita di ognuno. Non ritengo si possano dare lezioni a chicchessia sulla morte. A tal riguardo vorrei ricordare un passo di un noto saggio di Kant.

Nel 1791 Kant scrisse un saggio intitolato Sullinsuccesso di ogni saggio filosofico di teodicea. Il tema di fondo era come si possano conciliare l'esistenza del Male con gli attributi tradizionali di Dio, bontà ed onnipotenza. Kant conviene (in senso giuridico) la teodicea davanti al tribunale della filosofia. Osserva che l'avvocato della teodicea può replicare alle accuse che vengono mosse alla sua «assistita» (uso un termine processuale) con alcune affermazioni. In relazione alle proteste che vengono formulate alla «santità della volontà divina in nome del male morale», Kant scrive (punto $1^{\circ}$, lett. a) che l'avvocato della teodicea potrebbe obiettare che

la saggezza divina giudica secondo regole totalmente diverse e per noi inconcepibili, in base alle quali ciò che relativamente alla nostra ragion pratica e alla sua determinazione è giustamente riprovevole, è forse invece, relativamente ai fini divini e alla saggezza suprema, proprio il mezzo più adatto a promuovere il nostro particolare benessere come quello dell'universo in generale.

Nella ricostruzione della risposta, Kant fa riferimento alla frase «le vie dell'Essere Supremo non sono le nostre vie. Fin qui, nulla quaestio: Kant riferisce una risposta purtroppo ancor oggi abituale in certi ambienti. Infatti «liquida» la risposta della difesa della teodicea in modo lapidario:

Questa difesa, in cui la risposta è peggiore dell'accusa, non merita confutazione e può essere tranquillamente abbandonata all'esecrazione di ogni uomo, che abbia un ancorché minimo senso della moralità.

E tuttavia qualche pagina più avanti, Kant, trattando dei residui spazi che la teodicea (autentica) potrebbe avere dopo le sue critiche, ne indica uno nella risposta di Giobbe a Dio. Kant fa leva sulla sincerità di Giobbe, che è stato imprudente, ma non empio (mentre i suoi amici non han parlato con sincerità, ma per compiacere Dio). Eppure se si leggono i capitoli 38 e seguenti del libro di Giobbe, si noterà come in essi vi è la rivendicazione di Dio della sua superiorità e onniscienza, che l'uomo non può comprendere. E Giobbe accetta tale risposta. Insomma, alla fine, dal punto di vista esteriore, Giobbe dà la stessa risposta che Kant mette, stigmatizzandola, in bocca all'avvocato della teodicea.

Siamo al cospetto di una contraddizione di Kant? Probabilmente no: probabilmente ciò che il filosofo di Königsberg ci vuole dire è che solo chi prova una certa sofferenza può parlare della sofferenza. Della propria sofferenza. Può trarne insegnamenti per sé e può offrire la propria testimonianza in merito. Ma nemmeno lui è legittimato a porre gli insegnamenti che ne ha tratto, $\mathrm{i}$ principi regolatori della propria vita, come punto di riferimento assoluto, valido per tutti. Ecco perché rabbrividisco quando leggo frasi di questo tipo:

Tutto ciò che è contro la vita stessa, come ogni specie di omicidio, il genocidio, l'aborto, l'eutanasia e lo stesso suicidio volontario; $[. .$.$] tutte$ 
queste cose, e altre simili, sono certamente vergognose e mentre guastano la civiltà umana, ancor più inquinano sia coloro che così si comportano, che quelli che le subiscono; e ledono grandemente l'onore del Creatore ${ }^{4}$.

Ciò che lascia «disorientati» è l'accomunare fattispecie diversissime: omicidio, genocidio, aborto, eutanasia, suicidio. Ammesso per pura ipotesi (ma negato in tesi) che si tratti in ogni caso di fatti contro Dio, non è possibile accorparli senza distinzione e senza una graduazione della gravità e soprattutto non sembra possibile non distinguere tra fatti contro gli altri (o, addirittura, come nel caso del genocidio, contro l'umanità) e fatti contra se.

\section{Posizione del magistero ecclesiastico}

Nella Dichiarazione sull'eutanasia della Sacra Congregazione per la Dottrina della Fede (5 maggio 1980) ${ }^{5}$ - rivolta ai cristiani, ai seguaci di altre religioni e agli uomini di buona volontà, che «hanno tuttavia una viva coscienza dei diritti della persona umana» - suicidio ed eutanasia sono pratiche ritenute inaccettabili. Affermato che «per eutanasia s'intende un'azione o un'omissione che di natura sua, o nelle intenzioni, procura la morte, allo scopo di eliminare ogni dolore», la Sacra Congregazione ribadisce

con tutta fermezza che niente e nessuno può autorizzare l'uccisione di un essere umano innocente, feto o embrione che sia, bambino o adulto, vecchio, ammalato incurabile o agonizzante. Nessuno, inoltre, può richiedere questo gesto omicida per se stesso o per un altro affidato alla sua responsabilità, né può acconsentirvi esplicitamente o implicitamente. Nessuna autorità può legittimamente imporlo né permetterlo. Si tratta, infatti, di una violazione della legge divina, di un'offesa alla dignità della persona umana, di un crimine contro la vita, di un attentato contro l'umanità.

La Sacra Congregazione non ignora che la scelta di eutanasia può derivare dall'insopportabilità del dolore. Tuttavia, benché si premuri di precisare che in tali casi la responsabilità personale sia attenuata e forse anche non sussistente, la condanna del gesto resta ferma: «L'errore di giudizio della coscienza forse pure in buona fede - non modifica la natura dell'atto omicida, che in sé rimane sempre inammissibile».

Il paragrafo del documento dedicato all'eutanasia si conclude con la precisazione secondo cui «le suppliche dei malati molto gravi, che talvolta invocano la morte, non devono essere intese come espressione di una vera volontà di eutanasia; esse infatti sono quasi sempre richieste angosciate di aiuto e di affetto».

\footnotetext{
${ }^{4}$ Costituzione conciliare Gaudium et spes, $₫ 27$, lett. c (7dicembre 1965).

${ }^{5} \mathrm{La}$ si può leggere sul sito internet www.vatican.va.

${ }^{6}$ L'affermazione è oggetto di critiche. H. Kung, La dignità della morte. Tesi sull'eutanasia, Datanews Editrice, Roma 2007, p. 54, rileva come non si parli mai delle persone che si suicidano; e ancora precisa (pp. 77 ss.) che non solo non sempre gli analgesici possono alleviare il dolore, ma vi sono malati che desiderano morire per la perdita della dignità, del senso della vita, per mancanza di prospettive. «È vero - scrive Küng a p. 88 - che dietro qualche desiderio di mo-
} 
Ovviamente questo riferimento alla richiesta di aiuto implica la soluzione di due ulteriori questioni: da una parte quella relativa all'uso di analgesici, dall'altra quella della proporzionalità dei mezzi terapeutici.

Quanto alla prima, la Sacra Congregazione riafferma la legittimità dell'uso degli analgesici, ma con delle distinzioni. In primo luogo, si afferma legittimo il rifiuto degli analgesici da parte di coloro che ritengono in tal modo di partecipare alle sofferenze di Cristo. Inoltre, per quel che concerne l'uso degli analgesici che comportano la perdita della coscienza, si ribadisce l'insegnamento di Pio XII per il quale «non è lecito privare il moribondo della coscienza di sé senza grave motivo». Precisa al riguardo il documento che «è molto importante $[\ldots]$ che gli uomini non solo possano soddisfare ai loro doveri morali e alle loro obbligazioni familiari, ma anche e soprattutto che possano prepararsi con piena coscienza all'incontro con il Cristo»?

Quanto all'uso proporzionato dei mezzi terapeutici, la Sacra Congregazione, riaffermato che curarsi è un dovere, ammette: la liceità morale della rinuncia a terapie che si stanno rivelando inutili al trattamento del singolo; il rifiuto di terapie pericolose o onerose; la rinuncia alla prosecuzione di terapie «che procurerebbero soltanto un prolungamento precario e penoso della vita».

I criteri ispiratori del documento sono ripresi nell'enciclica di Giovanni Paolo II Evangelium vitae (25 marzo 1995) ${ }^{8}$.

\section{Note sul pensiero cristiano minoritario}

Rispetto le idee altrui e rispetto l'idea di un valore salvifico della sofferenza. Ritengo, tuttavia, che questa visione non si possa imporre. Valgono qui gli stessi principi che furono affermati e difesi quasi 40 anni fa in occasione del referendum sul divorzio.

rire sta soprattutto il desiderio di accompagnamento umano, di vicinanza e affetto. Ma è sbagliato, presuntuoso, voler squalificare ogni desiderio per partito preso, o come pretesto».

${ }^{7}$ Affermazione ripresa dall'enciclica Evangelium vitae citata alla nota successiva.

${ }^{8} \mathrm{La}$ si può leggere sul sito internet www.vatican.va. L'eutanasia è vista come il tentativo dell'uomo di «impadronirsi della morte, procurandola in anticipo e ponendo così fine "dolcemente" alla vita propria o altrui. In realtà, ciò che potrebbe sembrare logico e umano, visto in profondità si presenta assurdo e disumano. Siamo qui di fronte a uno dei sintomi più allarmanti della "cultura di morte", che avanza soprattutto nelle società del benessere, caratterizzate da una mentalità efficientistica che fa apparire troppo oneroso e insopportabile il numero crescente delle persone anziane debilitate. Esse vengono molto spesso isolate dalla famiglia e dalla società, organizzate quasi esclusivamente sulla base di criteri di efficienza produttiva, secondo i quali una vita irrimediabilmente inabile non ha più alcun valore» ( $\left.\int 64\right)$. Non sfuggirà la parziale visione che sottostà ad una tale durezza di giudizio, che vieppiù cresce nello svolgersi delle argomentazioni, fino a definire «falsa pietà»l'intento di evitare inutili sofferenze, ancora una volta esaltando la sofferenza: «Vivere per il Signore significa anche riconoscere che la sofferenza, pur restando in se stessa un male e una prova, può sempre diventare sorgente di bene. Lo diventa se viene vissuta per amore e con amore, nella partecipazione, per dono gratuito di Dio e per libera scelta personale, alla sofferenza stessa di Cristo crocifisso. In tal modo, chi vive la sua sofferenza nel Signore viene più pienamente conformato a lui $[. .$.$] e intimamente associato alla sua$ opera redentrice a favore della Chiesa e dell'umanità» (\$ 67). Mi sembra che non manchino da parte cattolica posizioni meno tranchantes: cfr. G. Piana, Testamento biologico, Cittadella Editrice, Assisi 2010, pp. 5 ss., e C.M. Martini - I. Marino, Credere e conoscere, Einaudi, Torino 2012, p. 69. Critiche alle argomentazioni papali sono state formulate dal valdese E. Genre, Dare dignità al morire, in AA.VV., Eutanasia. La legge olandese e commenti, a cura di P. Ricca, Claudiana, Torino 2002, pp. 47 ss., spec. p. 55. Secondo questo teologo, nell'enciclica si citerebbero passi biblici estrapolati dal loro contesto. 
Mi sembra che le Istituzioni ecclesiastiche adottino parole estremamente dure (e, a mio modesto avviso, fuorvianti) in relazione all'eutanasia (la quale, come si è visto, è definita addirittura "gesto omicida»). Fuorvianti, al di là della loro infondatezza: e ciò lo si può misurare dal fatto che l'eutanasia è pratica approvata da uno dei maggiori profeti della nonviolenza, Gandhi, il quale afferma:

Se mio figlio fosse contagiato dalla rabbia e non vi fosse nessun rimedio per alleviare la sua agonia, dovrei considerare mio dovere ucciderlo. Il fatalismo ha i suoi limiti. Noi lasciamo che il Fato compia il suo corso dopo aver esaurito tutti i rimedi. E il rimedio estremo per alleviare l'agonia di un bambino straziato è quello di togliergli la vita' .

Non mancano, peraltro, all'interno del mondo cristiano, posizioni di segno differente. Mi soffermerò in questa sede sulla testimonianza del teologo svizzero Hans Küng. Questi, dopo aver affermato l'assoluta esigenza di un'assistenza al malato terminale («non si sottolineerà mai abbastanza quanto sia importante per il malato incurabile una dedizione umana che duri fino alla fine» ${ }^{10}$ da parte dei medici, infermieri e parenti) osserva che:

Non possiamo però ignorare che oggi ci sono sempre più uomini e donne che non sopportano più una vita ormai perduta, $i$ cui indescrivibili dolori non scompaiano neppure con i più potenti sedativi $[\ldots]$. Costoro non desiderano essere tranquillizzati o resi incoscienti mediante psicofarmaci o morfina, con il rischio di privarsi del dialogo con i propri cari. Essi desiderano piuttosto congedarsi e morire in piena coscienza. Ma, dal momento che non possono morire, domandano una morte dignitosa: chiedono d'essere aiutati a morire ${ }^{11}$.

Ciò precisato, il teologo svizzero si preoccupa di chiarire che cosa sia l'eutanasia, sgomberando il campo d'indagine da alcuni equivoci ed ambiguità. Esistono, infatti, pratiche di costrizione alla morte, come il caso della soppressione di soggetti handicappati (circostanza purtroppo verificatasi sotto il regime nazista), che Küng definisce forme di «pseudo-eutanasia» (il che è quasi un eufemismo: in tali casi siamo al cospetto di azioni criminose sic et simpliciter ${ }^{12}$ ). Ciò che manca in tali casi, infatti, oltre alla volontà del «paziente», è l'insopportabile sofferenza fisica, che ci sembra elemento costitutivo della fattispecie dell'eutanasia, o, quantomeno, di una delle fattispecie di eutanasia (non si debbono dimenticare le sofferenze psichiche). Neppure mi sembra rientrino propriamente in tali fattispecie la semplice somministrazione di antidolorifici e la cosiddetta eutanasia passiva, «dove la morte è l'effetto collaterale [...] [del]l'interruzione dei mezzi di sostentamento artificiale della vita» ${ }^{13}$.

\footnotetext{
9 Il passo è tratto da un articolo del 18 novembre 1926 su Young India ed è riprodotto in M.K. GANDHI, Teoria e pratica della non violenza, Einaudi, Torino 1973, pp. 72 ss.

${ }^{10}$ H. KÜNG, op.cit., p. 32 (il corsivo è dell'A.).

${ }^{11}$ Ivi, p. 35.

${ }^{12}$ In tal senso cfr. anche C. Angelino nell'Introdurione al volume In difesa dell'eutanasia, cit., pp. 8 ss.

${ }^{13}$ H. KÜNG, op.cit., p. 38.
} 
L'eutanasia in senso stretto è quella attiva. Küng precisa: «Mi riferisco qui invece esclusivamente a casi di uomini che (vecchi o giovani che siano) si trovano alla fine della loro vita e vanno inesorabilmente incontro alla morte» ${ }^{14}$; e si domanda:

È lecito a questi uomini, giunti a questo stadio della loro vita, decidere di essa? Esiste un diritto - anche cristianamente responsabile all'autodeterminazione nel morire come nel vivere? Si può chiedere al medico anche un'eutanasia attiva? È lecito per il medico praticarla? ${ }^{15}$.

Küng ricorda che una risposta negativa a questa domanda è prevalentemente di ispirazione cristiana e si fonda sull'assunto secondo cui «all'uomo non è moralmente lecito disporre liberamente della propria vita» ${ }^{16}$. I teologi, in particolare, affermano che «la vita dell'uomo si fonda su un "si" di Dio all'uomo; essa è creazione e dono di Dio e perciò resta per principio sottratta alla facoltà umana di disporne liberamente» ${ }^{17}$. Ed è questo assunto che Küng prende in esame, dando atto, peraltro, che la sua riflessione sul tema è influenzata anche da una sua esperienza personale: la perdita del fratello oltre cinquant'anni fa per un tumore al cervello.

Küng ricorda le tesi affermate dal documento della Sacra Congregazione per la dottrina della fede e taglia corto:

Dietro tali argomenti (i cosiddetti "argomenti della sovranità di Dio") sta una falsa immagine di Dio, che si basa su alcuni passi biblici scelti arbitrariamente e interpretati letteralmente: secondo questa concezione, Dio sarebbe il sovrano, assolutamente libero, degli uomini suoi servi, il loro signore e padrone, dominatore, legislatore e giudice assoluto e, in fondo, anche il loro carnefice; non, invece, il padre dei deboli, dei sofferenti, dei perduti, che dona la vita all'uomo e si prende cura di lui come una madre amorosa, il Dio solidale dell'alleanza, che vuole avere nell'uomo, fatto a sua immagine, un partner libero e responsabile ${ }^{18}$.

E precisa, con un pregnante crescendo:

A partire da questo secondo concetto di Dio, il compito del teologo, verso i malati terminali non è quello di una spiritualizzazione mistificante del dolore né tantomeno di una sua finalizzazione pedagogica ("il purgatorio sulla terra"), ma, seguendo Gesù guaritore dei malati, quello di ridurre il più possibile ed eliminare il dolore, che certamente insegna all'uomo a pregare, ma anche in molti casi a imprecare. Ci sono addirittura dei teologi che hanno paura di una "società senza dolore" ma ci si chiede dove vivano. Altri spingono ad una "partecipazione alle sofferenze di Cristo", come se Gesù stesso si fosse dichiarato a favore delle

\footnotetext{
${ }^{14}$ Ivi, p. 41.

${ }^{15}$ Ibidem. Il corsivo nelle frasi riportate è proprio del testo originario.

${ }^{16}$ Ivi, p. 42.

${ }^{17}$ Ivi, p. 44.

${ }^{18}$ Ivi, p. 47. Cfr. anche la nota successiva.
} 
insopportabili sofferenze di un malato terminale, mantenuto in vita solo dagli artifici della medicina ${ }^{19}$.

La presa di distanza rispetto al documento del 5 maggio 1980 della Sacra Congregazione per la dottrina della fede qui è evidentissima!

Occorre, peraltro, sottolineare una fondamentale precisazione di Küng.

Non penso affatto - scrive il teologo - che basti una malattia incurabile, o gli acciacchi della vecchiaia, o una definitiva perdita della coscienza a ridurre l'uomo a una "non persona", a un "non più uomo" [...]. Il mio punto di vista è esattamente l'opposto: proprio perché l'uomo è nomo e resta tale fino alla fine anche quando è afflitto da una malattia incurabile (caso in cui la morte sia da attendere in un tempo comunque determinato) o quando è moribondo (caso in cui la morte sia imminente) egli ha diritto non solo ad una vita degna dell'uomo, ma anche ad una morte e ad una dipartita degna dell'uomo, e l'impiego di tecniche che lo mantengono in vita ad ogni costo, quando ormai "vita" può solo significare un'agonia di ore, mesi o anni e un'esistenza da vegetale rischia forse (dico "forse") di negargli questo diritto ${ }^{20}$.

Secondo Küng, sia l'inizio della vita umana sia la fine sono poste da Dio in mano alla responsabilità dell'uomo ${ }^{21}$. Egli ritiene possibile sul tema dell'eutanasia

una terza via teologicamente e cristianamente responsabile: tra un libertinismo antireligioso e irresponsabile ("diritto illimitato al suicidio") e un rigorismo reazionario senza compassione ("anche ciò che è insopportabile deve essere accolto come dono di Dio") $)^{22}$.

È la strada dell'autodeterminazione dell'uomo: «il diritto alla vita non equivale ad una coercizione a vivere» ${ }^{23}$. Siamo lontani da concezioni che vedevano in simili assunti una sorta di «tracotante ostinazione contro Dio» ${ }^{24}$.

\footnotetext{
${ }^{19}$ Ivi, p. 47. Sul punto vedasi anche il documento approvato dal Sinodo delle chiese valdesi e metodiste del 2000, in AA.VV., Eutanasia, cit., pp. 93 ss., spec. p. 96 in cui si legge: «Va respinta $[\ldots]$ l'idea di un valore intrinseco della sofferenza».

${ }^{20}$ Ivi, p. 48. I corsivi nel testo sono dell'A.

${ }^{21}$ Ivi, p. 52.

22 Ivi, p. 63.

23 Ivi, p. 54.

${ }^{24}$ Ivi, p. 63. Mi sembra opportune qui ricordare le parole, in chiave problematica, del teologo valdese P. Ricca, Vivere: un diritto o un dovere? Problematiche dell'eutanasia, saggio del 1986, ora ripubblicato in AA.VV., Eutanasia, cit., pp. 37 ss., spec. p. 40: «Ci si deve seriamente chiedere se una vita umana sottoposta a sofferenze inutili e disumanizzanti senza che ci sia alcuna possibilità di sbocchi positivi, abbia le caratteristiche del dono di Dio. Potrebbe dirsi che vi sia solo più la forma ma non più la sostanza del dono; potrebbe darsi che ci sia solo più l'apparenza ma non più la realtà del dono. E' vero, la vita umana è dono di Dio. Ma è ancora vita umana quella intorno alla quale si può formulare l'ipotesi dell'eutanasia? Non è forse una vita che ha perso proprio quelle caratteristiche personali che la rendono umana? Non ci sono forse momenti o situazioni in cui la vita umana diventa sub-umana o dis-umana così da essere irriconoscibile come dono di Dio? Momenti e situazioni in cui devo dire: no, questa non è più la vita che Dio ci ha dato, perché Dio fa doni buoni ai suoi figli, ma questo non è un dono buono, non viene da Lui, ma dalle forze del male e della distruzione. Quindi intervenendo su questa vita "larvale"
} 
Küng afferma di ritenere possibile l'eutanasia alle seguenti condizioni: 1) la richiesta della morte deve venire dal paziente stesso; 2) essa deve essere giustificata su una situazione di intollerabilità del dolore; 3) l'azione relativa dev'essere compiuta esclusivamente da un medico, previo consulto con altro medico $^{25}$.

\section{La legge olandese sull'eutanasia}

I punti individuati da Küng trovano un riscontro nella legge olandese sull'eutanasia, in vigore dal $1^{\circ}$ aprile $2002^{26}$, la cui denominazione ufficiale è «Legge sul controllo dell'interruzione della vita su richiesta e dell'assistenza al suicidio» (così testualmente nell'art. 24) ${ }^{27}$. In nuce questa legge prevede delle scriminanti rispetto ai reati di omicidio del consenziente (art. 293 cod. pen. $\mathrm{ol}^{28}$ ) e di aiuto al suicidio (art. $294 \mathrm{cod}$. pen. ol. ${ }^{29}$ )

quando [il fatto] sia stato commesso da un medico che si sia attenuto ai criteri di diligenza, prudenza e perizia di cui all'art. 2 della legge sul controllo dell'interruzione della vita su richiesta e dell'assistenza al suicidio [su cui tra breve riferiremo], e che ne abbia dato comunicazione al necroscopo comunale $[\ldots]$.

Il citato art. 2 prevede che i criteri di diligenza, prudenza e perizia che consentono al medico di poter praticare, secondo le regole di una buona pratica clinica, l'interruzione della vita o dare assistenza al suicidio al paziente che lo abbia chiesto, ricorrano laddove il medico:

a) abbia maturato la convinzione che si tratti di una richiesta spontanea, ben ponderata e definitiva del paziente; b) abbia maturato la convinzione che si tratti di una sofferenza insopportabile e senza speranza di miglioramento per il paziente; c) abbia informato il paziente della situazione in cui si trova e delle prospettive che ne derivano; d) abbia maturato, insieme al paziente, la convinzione che non esista alcun'altra soluzione ragionevole per la situazione in cui il paziente medesimo si era venuto a trovare; e) abbia consultato almeno un altro medico indipendente che abbia a sua volta visitato il paziente ed abbia espresso un pa-

\footnotetext{
io non spodesto Dio, non gli usurpo la sua signoria sulla vita (e sulla morte) ma blocco le potenze devastanti del male, pur soccombendo ad esse. Queste potenze vogliono farmi morire, bene, ma almeno morirò come voglio io (senza soffrire) e quando voglio io, anziché come e quando vogliono loro. In questo quadro l'eutanasia non è un atto di insubordinazione a Dio ma un atto di resistenza alla forza devastatrice del male. Ma allora, dire sì all'eutanasia non significa dire no a Dio ma semplicemente no al furore devastante e cieco e assurdo del male. Paradossalmente, l'eutanasia non smentisce la vita come dono di Dio, al contrario interviene proprio per evitare che questo dono diventi irriconoscibile come dono di Dio. L'eutanasia appare come mezzo estremo per preservare il volto umano della morte e quindi anche della vita. L'eutanasia è un aspetto del grande sforzo di umanizzazione della vita, che comprende anche l'umanizzazione della morte».

${ }^{25}$ H. Küng, op.cit., pp. 57 ss.

${ }^{26}$ Se ne può leggere il testo in AA.VV., Eutanasia, cit., pp. 9 ss.

${ }^{27}$ A quanto consta ripresa anche da legge belga approvata nel corso del 2002.

${ }^{28} \mathrm{La}$ norma trova una corrispondenza nel codice penale italiano all'art. 579.

${ }^{29}$ La norma trova una corrispondenza nel codice penale italiano all'art. 580.
} 
rere per iscritto circa il rispetto dei criteri di diligenza, prudenza e perizia di cui alle precedenti lettere da a) a d).

Lo stato d'incapacità del paziente non è ostativo alla pratica di interruzione della vita laddove, prima di entrare in tale stato, egli abbia rilasciato una dichiarazione scritta contenete la relativa domanda. minori.

Non entro in questa sede nella disamina delle disposizioni relative ai

È importante sottolineare che il medico deve relazionare il necroscopo comunale (art. 21 legge cit., che riforma la legge mortuaria), che trasmette la relazione ad una Commissione regionale di controllo ${ }^{30}$, la quale, ai sensi dell'art. $8,1^{\circ}$ comma, «accerta $[\ldots]$ se il medico, che ha praticato l'interruzione della vita su richiesta oppure ha dato assistenza al suicidio, abbia agito o meno in conformità ai criteri di diligenza, prudenza e perizia [...]». Laddove il giudizio sia negativo, la Commissione ne riferisce al P.M. e all'Ispettore regionale della sanità.

Mi sembra importante notare che la legge olandese affida la scelta dell'interruzione della vita esclusivamente ad una decisione tra medico e paziente. Nessuna autorità pubblica deve approvare la decisione prima che sia posta in pratica. In senso tecnico, nessuna autorità deve autorizzare l'interruzione della vita.

\section{La legge italiana}

Nel nostro Paese le cose sono assai articolate. Dal punto di vista penalistico vi sono due norme che sembrano ostative ad ogni sviluppo.

L'art. 579 c.p. dispone infatti: «Chiunque cagiona la morte di un uomo, col consenso di lui, è punito con la reclusione da sei a quindici anni» (in Olanda la pena edittale è fino a dodici anni). Si noti che nel $3^{\circ}$ comma, detto articolo prevede che la pena sia quella del delitto d'omicidio (reclusione non inferiore ad anni ventuno) «se il fatto è commesso: [...] 2) contro una persona inferma di mente, o che si trova in condizioni di deficienza psichica, per un'altra infermità o per l'abuso di sostanze alcoliche o stupefacenti». Si pensi, quindi, a quali ostacoli, de iure condito, in virtù di questa norma, vi sono all'eutanasia. Dal canto suo l'art. 580 c.p.c. prevede che «chiunque determina altri al suicidio o rafforza l'altrui proposito di suicidio, ovvero ne agevola in qualsiasi modo l'esecuzione, è punito, se il suicidio avviene, con la reclusione da cinque a dodici anni». Evidenti, in tal caso, anche le difficoltà rispetto al suicidio assistito ${ }^{31}$.

E tuttavia non sono mancate revisioni nell'interpretazione della portata della norma, anche alla luce del disposto dell'art. 32 Cost. e di convenzioni internazionali ${ }^{32}$.

L'art. $32,2^{\circ}$ comma, Cost. sancisce che «nessuno può essere obbligato a un determinato trattamento sanitario se non per disposizione di legge». Così

\footnotetext{
${ }^{30}$ Della Commissione debbono far parte, ai sensi dell'art. 3, $2^{\circ}$ comma, 1. cit. almeno un esperto in materie giuridiche, che la presiede, un medico e un esperto in questioni etiche o in questioni attinenti la tutela dei diritti fondamentali.

31 In Olanda l'istigazione al suicidio è fattispecie differente da quella dell'aiuto al suicidio: la prima è disciplinata nel $1^{\circ}$ comma dell'art. 294; la seconda nel $2^{\circ}$ comma dello stesso articolo. La pena detentiva è per entrambe fino a tre anni: ma l'esimente introdotta dalla legge in esame è, ovviamente, correlata solo all'aiuto al suicidio.

32 Sul punto cfr. S. Rodotà, La vita e le regole, Feltrinelli, Milano 2006, pp. 250 ss.
} 
pure l'art. 33, $1^{\circ}$ comma, della L. 23 dicembre 1978, n. 833 dispone che «gli accertamenti ed i trattamenti sanitari sono di norma volontari». Più recentemente, poi, la Convenzione di Oviedo (che è legge dello Stato italiano), all'art. 5 prevede che

une intervention dans le domaine de la santé ne peut être effectuée qu'après que la personne concernée y a donné son consentement libre et éclairé. //Cette personne reçoit préalablement une information adéquate quant au but et à la nature de l'intervention ainsi que quant à ses conséquences et ses risques. // La personne concernée peut, à tout moment, librement retirer son consentement ${ }^{33}$.

A tale disposizione fa riscontro il disposto dell'art. 9 della Convenzione secondo cui «les souhaits précédemment exprimés au sujet d'une intervention médicale par un patient qui, au moment de l'intervention, n'est pas en état d'exprimer sa volonté seront pris en compte».

Sotto quest'ultimo aspetto occorre precisare che la mancanza di disposizioni scritte non impedisce la ricostruzione della volontà del paziente che si trovi in stato vegetativo permanente. È sulla base di queste norme che, secondo Cass., 16 ottobre 2007, n. 21748 (caso Englaro) ${ }^{34}$,

ove il malato giaccia da moltissimi anni [...] in stato vegetativo permanente, con conseguente radicale incapacità di rapportarsi al mondo esterno, e sia tenuto artificialmente in vita mediante un sondino nasogastrico che provvede alla sua nutrizione ed idratazione, su richiesta del tutore che lo rappresenta, e nel contraddittorio con il curatore speciale, il giudice può autorizzare la disattivazione di tale presidio sanitario (fatta salva l'applicazione delle misure suggerite dalla scienza e dalla pratica medica nell'interesse del paziente), unicamente in presenza dei seguenti presupposti: (a) quando la condizione di stato vegetativo sia, in base ad un rigoroso apprezzamento clinico, irreversibile e non vi sia alcun fondamento medico, secondo gli standard scientifici riconosciuti a livello internazionale, che lasci supporre la benché minima possibilità di un qualche, sia pure flebile, recupero della coscienza e di ritorno ad una percezione del mondo esterno; e (b) sempre che tale istanza sia realmente espressiva, in base ad elementi di prova chiari, univoci e convincenti, della voce del paziente medesimo, tratta dalla sue precedenti dichiarazioni ovvero dalla sua personalità, dal suo stile di vita e dai suoi convincimenti, corrispondendo al suo modo di concepire, prima di cadere in stato di incoscienza, l'idea stessa di dignità della persona. Ove l'uno o l'altro presupposto non sussista, il giudice deve negare l'autorizzazione, dovendo allora essere data incondizionata prevalenza al diritto alla vita, indipendentemente dal grado di salute, di autonomia

\footnotetext{
${ }^{33}$ Sul punto si vedano le considerazioni che nel 1985 aveva in tal senso espresso H. Jonas, Il diritto di morire, il melangolo, Genova 1991, pp. 22 ss.

${ }^{34} \mathrm{La}$ sentenza è pubblicata nelle maggiori riviste giuridiche. Cfr., per tutte, "Foro Italiano», 2008, I, 2609 con nota di S. Cacace. Su detta sentenza vedi anche A. Santosuosso, La salute tra scienza e libertà, in «Questione Giustizia», 2011, 3-4, pp. 219 ss., spec. pp. 224 ss. 
e di capacità di intendere e di volere del soggetto interessato e dalla percezione, che altri possano avere, della qualità della vita stessa.

\section{La giurisprudenza della Cedu}

Ma non si dimentichino le pronunce della Corte europea dei diritti dell'uomo ${ }^{35}$. L'orientamento di queste sentenze non è univoco.

Noto è il caso Pretty del 29 aprile 2002. La sig.ra Pretty era malata di sclerosi laterale amiotrofica (SLA). Nel luglio 2001 richiede al Direttore del Public Prosecutions di impegnarsi a non perseguire suo marito laddove quest'ultimo, aderendo al suo (della moglie) desiderio, l'avesse aiutata a suicidarsi.

Il rifiuto dell'Ufficio e la successiva vicenda giudiziaria hanno avuto sbocco a Strasburgo per violazione di diverse norme della Convenzione sui diritti dell'uomo. In particolare la sig.ra Pretty denunciava la violazione ai sensi di cinque norme della Convenzione. I motivi più rilevanti sono quelli fondati sugli artt. 2 e $8^{36}$. La Corte respinse all'unanimità il ricorso della sig.ra Pretty, verso la quale non mancò di esprimere parole di solidarietà e compassione.

Riguardo alla violazione dell'art. 2 della Convenzione, la Corte affermò ( $(39)$ che esso «non ha alcun rapporto con le questioni concernenti la qualità della vita o ciò che una persona sceglie di fare della sua vita [...]. L'art. 2 non potrebbe, senza distorsione del tenore letterale, essere interpretato come conferente un diritto diametralmente opposto, ossia un diritto a morire». Di qui la conclusione che non sia possibile dedurre dall'art. 2 della Convenzione un diritto a morire, per mano di un terzo o con l'assistenza di una pubblica autorità $(\mathbb{\Omega}$ 40).

Riguardo alla violazione dell'art. $8,1^{\circ}$ comma, la Corte ritenne infondata la questione, pur ammettendo che «la dignità e la libertà dell'uomo sono l'essenza stessa della Convenzione» (\$ $(55)$ anche sotto il profilo della qualità della vita. Nondimeno, la Corte affermò che non vi è ingerenza dello Stato nella vita delle persone con la previsione legislativa che punisce il suicidio assistito. Lo Stato deve intervenire a tutela dei più deboli e vulnerabili. È vero - sostenne la Corte - che il caso dei malati terminali ha una sua specificità, ma incombe agli Stati di valutare i rischi di abusi connessi ad eccezioni alla regola.

Sennonché dal 2002 qualcosa è cambiato. Nella sentenza del 20 gennaio 2011 (caso Haas v. Suisse), la Corte rigettò il ricorso del sig. Hass, persona affetta da gravi turbe psichiche, che chiedeva di poter avere la sostanza per

\footnotetext{
${ }^{35}$ Le sentenze della CEDU possono essere rintracciate comodamente nel sito della Corte stessa.

36 Per comodità del lettore riporto qui le relative norme. L'art. 2 dispone: «1. Il diritto alla vita di ogni persona è protetto dalla legge. Nessuno può essere intenzionalmente privato della vita, salvo che in esecuzione di una sentenza capitale pronunciata da un tribunale, nei casi in cui il delitto sia punito dalla legge con tale pena. 2. La morte non è considerata inflitta in violazione di questo articolo quando derivasse da un ricorso alla forza reso assolutamente necessario: a) per assicurare la difesa di qualsiasi persona da una violenza illegittima; b) per eseguire un arresto legale o per impedire l'evasione di una persona legalmente detenuta; c) per reprimere, in modo conforme alla legge, una sommossa o una insurrezione». Art. 8: «1. Ogni persona ha diritto al rispetto della sua vita privata e familiare, del suo domicilio e della sua corrispondenza. 2. Non può esservi ingerenza della pubblica autorità nell'esercizio di tale diritto a meno che tale ingerenza sia prevista dalla legge e costituisca una misura che, in una società democratica, è necessaria per la sicurezza nazionale, l'ordine pubblico, il benessere economico del paese, la prevenzione dei reati, la protezione della salute o della morale, o la protezione dei diritti e delle libertà altrui».
} 
l'iniezione letale senza prescrizione medica. Egli non aveva trovato medici disposti a prescrivergli il farmaco per l'iniezione letale; probabilmente i medici ritenevano che nel suo caso non ricorressero le condizioni per il suicidio assistito, non perché la malattia fosse di carattere psichico e non fisico, ma perché ritenevano che la malattia fosse suscettibile di trattamento medico (\$ 18). Il sig. Haas fece ricorso alla Corte dei diritti dell'uomo, assumendo che vi fosse nella fattispecie un'ipotesi di violazione dell'art. 8 della Convenzione sui diritti dell'uomo. La Corte affermò di ritenere che

il diritto di un individuo a decidere in quale maniera e in quale momento la sua vita debba essere chiusa, a condizione che egli sia in grado di formare liberamente la sua volontà e il suo proposito d'agire in conseguenza, è uno degli aspetti della sua vita privata ai sensi dell'art. 8 della Convenzione ( $(51)$.

Tuttavia il problema nella causa Haas non era questo, ma il diritto di avere la sostanza letale, senza prescrizione medica. E su questo punto la Corte ritenne che la domanda del sig. Haas fosse infondata, poiché ai sensi dell'art. 8, $2^{\circ}$ comma, lo Stato deve «impedire ad un individuo di mettere fine ai suoi giorni se la sua decisione non è stata presa liberamente e con piena cognizione di causa» (\$54). Dunque, col caso Haas, la Corte europea dei diritti dell'uomo ba corretto ilproprio orientamento.

Come si è visto, nel caso Pretty del 2002 la Corte affermò che l'art. 2 «non ha alcun rapporto con le questioni concernenti la qualità della vita o ciò che una persona sceglie di fare della sua vita [...]. L'art. 2 non potrebbe, senza distorsione del tenore letterale (langage), essere interpretato come conferente un diritto diametralmente opposto, ossia (à savoir) un diritto a morire». Di qui la conclusione che non sia possibile dedurre dall'art. 2 della Convenzione un diritto a morire, per mano di un terzo o con l'assistenza di una pubblica autorità $(\mathbb{S}$ 40). Con la stessa sentenza, pur ritenendo la Corte, in relazione all'art. 8, che «la dignità e la libertà dell'uomo sono l'essenza stessa della Convenzione» (\$ 65) anche sotto il profilo della qualità della vita, non si pervenne tuttavia all'affermazione di un diritto all'assistenza al suicidio dell'infermo sofferente. Ma con la sentenza Haas qualcosa comincia a cambiare. Non si afferma - certo - che gli Stati membri debbano comunque prevedere un procedimento di assistenza al suicidio (tra l'altro la Svizzera, Stato convenuto nel procedimento Haas, ammette tale procedimento), ma si afferma che

il diritto di un individuo a decidere in quale maniera e in quale momento la sua vita debba essere chiusa, a condizione che egli sia in grado di formare liberamente la sua volontà e il suo proposito d'agire in conseguenza, è uno degli aspetti della sua vita privata ai sensi dell'art. 8 della Convenzione ( $(51)$.

Un ulteriore passo in avanti nel riconoscimento del diritto a morire è stato compiuto nel luglio 2012 con la sentenza Koch.

La sig.ra Koch a 52 anni, a seguito di una caduta per le scale, restò tetraplegica. Aveva problemi di autonomia respiratoria e spasmi. La prognosi medica era comunque di una quindicina di anni di vita. La signora Koch e- 
spresse peraltro il desiderio di mettere fine a quella che le pareva una vita indegna. Interpellò l'organizzazione svizzera Dignitas. Nel frattempo, chiese all'Istituto federale dei prodotti farmaceutici di poter avere 15 grammi di una sostanza per un'iniezione letale. L'autorizzazione fu rifiutata sul presupposto che la somministrazione dei farmaci è finalizzata al mantenimento o al prolungamento della vita, e non a consentire ad una persona di mettere fine ai suoi giorni ( $(10)$. Avverso detto rifiuto, la sig.ra Koch e suo marito proposero ricorso in via amministrativa. Nemmeno un mese dopo, tuttavia, si rese possibile il procedimento del suicidio assistito presso l'associazione svizzera Dignitas (12 febbraio 2005).

L'azione promossa dai sigg. Koch, peraltro, non si fermò. Il ricorso in via amministrativa fu respinto e, contro tale decisione, il sig. Koch propose azione giudiziaria. La vicenda giudiziaria fu portata fino alla Corte costituzionale federale, che, tuttavia, si limitò ad affermare la carenza di legittimazione attiva del sig. Koch. Questi adì, quindi, la Corte di Strasburgo. Ricorso articolato, poiché il sig. Koch lo fondò sugli artt. 6 (diritto ad un processo equo) e 8 della Convenzione, sia come erede della moglie, sia in proprio. E se effettivamente la Corte dei diritti dell'uomo ritenne che il sig. Koch non potesse agire per la moglie, per il carattere personalissimo dei diritti fatti valere, tuttavia, ritenne che una violazione dell'art. 8 fosse stata commessa dallo Stato membro (la Germania) per non aver pronunciato nel merito sulla richiesta dei coniugi ( $(\$ 72)$ e ciò avesse coinvolto anche la vita privata del sig. Koch, per lo stretto rapporto che aveva con la malata. Si ritenne quindi assorbita ogni questione relativa alla violazione dell'art. 6.

Tanto è ciò che si è tradotto nel dispositivo della sentenza ${ }^{37}$. Prendiamo comunque atto che la Corte di Strasburgo ha voluto fare un passo in più. Vedremo prossimamente che cosa accadrà col caso Alda Gross v. Suisse.

\section{Il caso Welby}

Vorrei ora soffermarmi sul caso Welby. Riassumo qui in breve la vicenda, seguendo la ricostruzione che ho reperito in alcuni articoli di dottrina ${ }^{38}$.

\footnotetext{
${ }^{37}$ Devo, peraltro, segnalare che nel corpo della motivazione, al $\int 54$, la Corte disse qualcosa di più, ritenendo che anche la decisione dell'Istituto federale per i farmaci di rigettare la domanda della sig.ra Koch avesse violato l'art. 8. E ciò fu affermato alla fine di una disamina dei precedenti della Corte stessa (Pretty e Haas), sottolineando (al $\int 51$ ) che nel primo di detti casi si era affermato che la Corte, al $\ 67$, non poteva escludere che il fatto di impedire per legge alla ricorrente di esercitare la sua scelta di evitare ciò che, ai suoi occhi, costituiva una fine indegna e penosa rappresenta una violazione al diritto dell'interessata al rispetto della sua vita privata ai sensi dell'art. 8, comma $1^{\circ}$ della Convenzione». La Corte - nel caso Koch - citando il caso Pretty, riporta tra virgolette la frase del $\int 67$. Ma la citazione è errata. Non solo la frase non corrisponde esattamente (ma questo è il meno: può accadere che in una citazione si renda un concetto anche se in una costruzione un po' diversa), ma il problema è che nel caso Koch la Corte ha isolato quel $\int 67$ del caso Pretty dal contesto: ha addirittura omesso la conclusione di quel paragrafo, in cui si legge: la Corte «esaminerà qui di seguito la questione per sapere se questa violazione è conforme alle esigenze del secondo comma dell'art. 8». Quel paragrafo, dunque, rientra in un contesto più ampio: la Corte affermò di non poter escludere, intendendo dire di non poterlo fare a priori. Ma poi finì per pervenire - seppure in modo sofferto e problematico - ad una conclusione diversa. Ciò lascia perplessi. Non è la prima volta che a livello di Corti apicali (o quasi) si risolvono in tal modo mutamenti di orientamenti. Tuttavia, il modo adottato in questo caso non mi sembra accettabile.

${ }^{38}$ Cfr. M. Azzalini, Trattamenti life-saving e consenso del paziente: $i$ doveri del medico dinanzi al rifiuto di cure, in «Nuova Giurisprudenza Civile Commentata», 2008, I, pp. 73 ss.
} 
Nel 1963 a Piergiorgio Welby, allora diciottenne, fu diagnosticata una distrofia muscolare scapolo-omerale progressiva, dall'esito certamente infausto. Nel corso degli anni ottanta, egli perse l'uso delle gambe; nel corso degli anni novanta, poi, ebbe un progressivo deterioramento del fisico, finché nel luglio del 1997 manifestò anche un'insufficienza respiratoria. Da quel momento Welby visse tracheotomizzato; perse la capacità di alimentarsi, di respirare e, sostanzialmente, di parlare in maniera comprensibile: verrà nutrito con un prodotto speciale, respirerà con l'ausilio di un apparato di ventilazione polmonare Exole3XO, comunicherà attraverso un computer.

Nel corso del 2006 Welby presentò al Tribunale di Roma un ricorso per provvedimento d'urgenza, con cui chiese che

accertato e dichiarato il [suo] diritto [...] ad autodeterminarsi nella scelta delle terapie mediche invasive alle quali sottoporsi e, quindi, il [suo] diritto [...] di manifestare il proprio consenso a taluni trattamenti ed il rifiuto ad altri; preso atto ed accertato altresì che [egli] [...] ha espresso, e ribadisce $[. .$.$] la propria libera, informata, consapevole ed incondizio-$ nata volontà a che sia immediatamente cessata l'attività sulla propria persona di sostentamento a mezzo di ventilatore artificiale mentre sia proseguita e praticata la terapia di sedazione terminale; [fosse] ordinato [...] [ai] soggetti che [lo] hanno in cura [...], di procedere all'immediato distacco del ventilatore artificiale che [gli] assicura la respirazione assistita $[\ldots]$, contestualmente somministrando[gli] [...] terapie sedative che, in conformità con le migliori ed evolute pratiche e conoscenze medico-scientifiche, risultino [a lui] idonee a prevenire e/o eliminare qualsiasi stato di sofferenza fisica e/o psichica [...].

Il Tribunale di Roma, con un provvedimento che lascia effettivamente disorientati, rigettò la richiesta ritenendo che sebbene

in linea di principio nell'ordinamento giuridico italiano può configurarsi il diritto del paziente alla consapevole ed informata autodeterminazione nella scelta delle terapie cui sottoporsi, ivi compresa quella di interrompere le terapie in corso, pur se l'esito è del tutto verosimilmente letale (ed anzi anche se tal esito è voluto dal paziente), tuttavia tale diritto non è in concreto tutelabile, a causa della mancata definizione, in sede normativa, delle sue modalità attuative, in particolare con riferimento all'esatta individuazione del c.d. divieto di accanimento terapeutico ${ }^{39}$.

Non è il caso di soffermarsi in una critica a tale motivazione. È facile convenire col Giudice penale, poi investito della vicenda, che:

Quando si riconosce l'esistenza di un diritto di rango costituzionale, quale quello all'“autodeterminazione individuale e consapevole" in materia di trattamento sanitario, non è, poi, consentito lasciarlo senza tutela, rilevandone, in assenza di una normativa secondaria di specifico riconoscimento, la sua concreta inattuabilità sulla scorta dell'esistenza di

\footnotetext{
39 Trib. Roma, 16 dicembre 2006, in «Il Corriere del Merito», 2007, pp. 461 ss., con nota di G.
} Casaburi. 
disposizioni normative di fonte gerarchica inferiore a contenuto contrario (omissis). In realtà, se si accogliesse una tale conclusione, potremmo incorrere in una palese violazione dei principi che presiedono alla disciplina della gerarchia delle fonti, in quanto non è consentito disattendere l'applicazione di una norma costituzionale sulla scorta dell'esistenza di norme contrastanti di valore formale inferiore, perché delle due è l'una: o si privilegia l'interpretazione che faccia salvo il principio costituzionale con immediata applicazione di quest'ultimo, disattendendo l'interpretazione contraria della norma, (omissis) oppure, in caso di insuperabile conflitto, si deve sollevare questione di legittimità costituzionale, ma certamente non si può lasciare inattuato un principio costituzionale e senza tutela giuridica il diritto soggettivo che da esso discende ${ }^{40}$.

Welby a questo punto si rivolse al dott. Riccio, che si dichiarò disponibile ad aiutarlo.

Imputato del reato di omicidio del consenziente (imputazione coattiva, disposta dal G.i.p.), il dott. Riccio fu assolto con una sentenza la cui massima è la seguente:

Non risponde di omicidio del consenziente, per aver agito nell'adempimento di un dovere, il medico che sospende il trattamento sanitario che tiene in vita il paziente, su richiesta consapevole di questi, costituendo tale richiesta esercizio di un diritto di rifiutare le cure (art. $32,2^{\circ}$ comma, Cost.) - cui corrisponde un divieto di attuare o proseguire gli interventi non voluti - prevalente sul diritto alla vita, quand'anche si intendesse quest'ultimo come "indisponibile". Considerata la lettera e la ratio dell'art. $32,2^{\circ}$ comma, Cost., assume però rilievo scriminante unicamente la richiesta di sospensione di cure rivolta ad un medico nel contesto di una rapporto sanitario stricto sensu; soltanto un medico, infatti, in quanto soggetto dotato di specifiche competenze e vincolato a precisi doveri deontologici, può garantire il rispetto dei confini tra l'esercizio di una libera ed informata autodeterminazione del paziente ed arbitrii forieri di violazioni di diritti essenziali, quali quelli in gioco $^{41}$.

\section{Progetti di legge italiani e conclusione}

E veniamo ai progetti di legge presentati al nostro Parlamento.

Nel corso delle ultime tre legislature sono stati presentati vari disegni di legge aventi per oggetto la «disciplina dell'eutanasia e del testamento biologico». In alcuni casi (ad es., nel corso della XV legislatura, la proposta di legge dei deputati Grillini, Belillo, Turci e Turco, contraddistinta col n. 1702) si è contemplata anche la previsione dell'eutanasia passiva.

In generale, i testi prevedono il dovere informativo del medico; disciplinano il consenso informato e le modalità della relativa prestazione; introducono la figura del testamento biologico, prevedendo in particolare che il testamento possa contenere la volontà espressa della persona: a) di rifiutare qualsiasi forma di rianimazione o di prolungamento dell'esistenza dipendente da appa-

${ }^{40}$ Trib. Roma, 17 ottobre 2006, in «Diritto penale e processo», 2008, pp. 59 ss., spec. p. 60.

41 Trib. Roma, 17 ottobre 2007, cit. 
recchiature, o alcune forme di rianimazione specifiche e definite, o di non essere sottoposta ad alcun trattamento terapeutico; b) di non essere sottoposta all'alimentazione o all'idratazione artificiali o per mano di terzi; c) di poter fruire, in caso di sofferenze anche psicologiche ritenute personalmente inaccettabili, degli opportuni trattamenti analgesici, anche qualora gli stessi possano provocare una riduzione della propria aspettativa di vita; d) di rifiutare specifici trattamenti, anche quando le condizioni di salute non sono definite critiche e di optare invece per trattamenti alternativi.

Per quanto concerne l'eutanasia si prevede che ogni persona che versi in condizioni terminali o che sia affetta da una patologia gravemente invalidante, irreversibile e con prognosi infausta, abbia diritto di porre termine alla propria esistenza mediante l'assistenza di un medico. Si precisa che una persona versa in condizioni terminali quando si trova in uno stato patologico incurabile cagionato da lesioni o da malattie e dal quale, secondo cognizione medicoscientifica, consegue la inevitabilità della morte, il cui momento sarebbe soltanto ritardato ove si facesse ricorso a terapie di sostentamento vitale utilizzando tecniche meramente rianimative nonché apparecchiature meccaniche o artifici per sostenere, riattivare o sostituire una funzione vitale naturale.

Quanto all'eutanasia attiva, si dispone che il medico curante che pratica l'eutanasia attiva non è punibile laddove la pratica di interruzione della vita avvenga in presenza delle seguenti condizioni: a) il paziente è maggiorenne e capace di intendere e di volere al momento della richiesta; b) la richiesta è stata formulata in maniera volontaria, è stata ben ponderata e ripetuta e non è il risultato di una pressione esterna; c) il paziente versa in condizioni terminali e le sue sofferenze fisiche o psichiche sono persistenti, insopportabili e tali da non poter essere alleviate da ulteriori trattamenti terapeutici.

Segue una disciplina dell'informazione che il medico deve fornire al paziente e delle procedure che deve rispettare, in linea di massima, sulla falsariga di quanto si è visto nella legge vigente in Olanda. È peraltro previsto l'obbligo di informazione dei prossimi congiunti e altre persone indicate dal paziente. Si precisano le formalità della dichiarazione che il paziente dovrebbe rilasciare, sancendo esplicitamente il principio della revocabilità della decisione.

Le proposte di legge prevedono l'istituzione di una Commissione nazionale di controllo, ma, a differenza della legge olandese, non si prevede esplicitamente l'informazione al P.M. nel caso di accertata violazione delle procedure previste dalla legge; tale obbligo è però previsto in via generale dall'art. 331 c.p.p.

In senso analogo alla legge olandese è da registrare che le proposte di legge affidano la scelta dell'interruzione della vita esclusivamente ad una decisione tra medico e paziente.

A differenza della legge olandese, alcune proposte hanno previsto e disciplinato anche un'ipotesi di eutanasia passiva. L'art. 7 della proposta di legge Grillini, Belillo, Turci e Turco (XV legislatura), al $2^{\circ}$ comma, stabiliva, infatti, che

l'accertamento delle condizioni terminali del paziente che abbia perduto la capacità naturale o la facoltà di comunicare è effettuato da un medico competente nelle tecniche di rianimazione su concorde parere del primario anestesiologico. Il medico che ha effettuato l'accertamento ne 
comunica i risultati alle persone che sono legittimate a proporre opposizione [n.d.e.: in linea di sommaria enunciazione, i parenti prossimi ed i conviventi del paziente] [...] e che sono agevolmente reperibili. Se non è accertata alcuna opposizione, e se il paziente non ha espresso personalmente e consapevolmente, nel testamento biologico [...] il consenso alle terapie di sostentamento vitale [...], il medico dispone per iscritto l'interruzione delle medesime terapie.

Insomma, se non erro, si ribaltava il principio di presunzione della scelta della vita. In determinati casi, cioè, ricorrendo le condizioni di vita terminale, si prevedeva che laddove il paziente non potesse esprimersi e laddove non ci fosse l'espressione ex ante di una sua volontà contraria e non vi fosse opposizione di prossimi congiunti, l'interruzione delle terapie di sostentamento era ${ }^{42}$ disposta. Tale previsione - se non erro - non risulta riprodotta in proposte della XVI legislatura.

Di regola è prevista l'obiezione di coscienza.

Non è il caso in questa sede di diffondersi sui dubbi interpretativi che possono nascere da alcuni tecnicismi dei testi. Qui basta aver delineato le linee di fondo di proposte, il cui iter parlamentare, peraltro, è stato interrotto ancora una volta dalla fine anticipata della legislatura.

Qui si chiude questo brevissimo excursus sulla questione dell'eutanasia.

Ciò che mi sembra di dover ribadire è l'autonomia della persona, la sua capacità di decidere autonomamente della sua vita e della sua morte, senza imposizioni che gli vengano da chicchessia, in nome di ideologie, di valori e di fedi che possono valere individualmente, ma non possono essere imposte alle generalità dei cittadini.

Ancora una volta ciò che viene in rilievo è anche la laicità dello Stato, che è principio supremo del nostro ordinamento giuridico.

\footnotetext{
${ }^{42}$ La proposta di legge non diceva «può», ma «dispone»: il che farebbe pensare quasi ad una sorta di obbligo, salva l'ipotesi dell'obiezione di coscienza che era contemplata dall'art. 8. 